\title{
Sistema electoral web para el Instituto Superior Tecnológico Luis Tello del cantón Esmeraldas
}

John Simisterra1

1 Instituto Superior Tecnológico Luis Tello, Esmeraldas, Ecuador 1jwsimisterra@istluistello.edu.ec

\section{RESUMEN}

Este artículo está basado en diseñar un sitio web para el proceso de elecciones estudiantiles del Instituto Superior Tecnológico Luis Tello (ISTLT) del cantón Esmeraldas; aquí se detallan solo procesos de estructuración y diseño del sistema, más no de funcionalidad mediante líneas de codificación. Primeramente se utilizó un proceso bibliográfico para sustentar el uso actual de la comunicación y la seguridad de la información online en los procesos electorales. Para diseñar las interfaces y base de datos del sitio se aplicó un modelamiento incremental agregando funcionalidades paulatinas en los refinamientos sucesivos realizados al sistema como las páginas mediante Google Sites, herramienta que aunque es gratuita y fácil de utilizar, presenta limitaciones para el desarrollo de sitios webs de alto nivel de profesionalismo. El sistema es confiable y ofrece una interfaz amigable en el manejo masivo de información online, cumpliendo la postura de seguridad de sitios web creados de forma exclusiva frente a la suspicaz credibilidad del manejo masivo de información online.

Palabras Clave: Elecciones por Internet, Sistema Electoral Web, Sufragio Online.

Abstract. This article is based on designing a website for the student elections process of the Luis Tello Higher Technological Institute (ISTLT) of the Esmeraldas canton; here only structuring and system design processes are detailed, but not functionality through coding lines. First, a bibliographic process was used to support the current use of communication and information security online in electoral processes. To design the interfaces and database of the site, an incremental modeling was applied adding gradual functionalities in the successive refinements made to the system such as pages through Google Sites, a tool that although it is free and easy to use, has limitations for the development of websites high level of professionalism. The system is reliable and offers a friendly interface in the massive handling of online information, complying with the security posture of websites created exclusively against the suspicious credibility of the massive handling of online information.

Key Words: Internet Elections, Web Electoral System, Online Suffrage. 


\section{Introducción}

Generalmente las instituciones de educación superior establecen periódicamente elecciones de representantes estudiantiles y el instituto del cantón Esmeraldas no es la excepción. En tal virtud, el instituto citado cuenta con un sistema electoral desarrollado de forma exclusiva que funciona mediante una red local en donde un computador hace las veces de servidor y los demás de clientes. Dicho sistema se adecuó a los procesos de votaciones presenciales, sin embargo debido a las restricciones de aglomeración ocasionadas por la pandemia suscitada por el covid-19 el uso de este sistema no es aplicable para procesos de elecciones actuales, además la virtualidad y la conectividad permanente se está transformando en una constante que utilizan las personas para estar continuamente comunicados entre sí y tener la información que requieran a la distancia de un clic.

El uso de plataformas online que de una u otra forma se vinculan en la participación de procesos electorales fue puesto en manifiesto en España según lo sostiene [1], en donde sus campañas electorales regionales del 2015 y 2016 hicieron uso de las redes sociales para ampliar la comunicación de sus propuestas políticas. Similar caso se presentó en Ecuador desde el año 2017 donde las redes sociales no solo compitieron con los medios de comunicación tradicionales, sino que propiciaron formas alternativas en la transmisión de información política [2].

En virtud de lo mencionado, se plantea la siguiente hipótesis ¿la implementación de un sitio web brindará la eficacia necesaria de comunicación en el proceso de sufragio de las elecciones estudiantiles del instituto de Esmeraldas?

Puesto que el uso de internet se ha convertido en parte casi imprescindible del vivir cotidiano de los seres humanos y goza de un papel muy importante en el desarrollo de las actividades que se realizan [3] la implementación de un sitio web permitirá que los estudiantes tengan la libertad de ser partícipes del proceso ejerciendo su voto desde el lugar y comodidad donde se encuentren mediante su dispositivo conectado al internet. Por ello, el presente proyecto tiene como propósito la implementación de un sistema electoral online mediante el uso de herramientas de diseño y programación web para las jornadas de elecciones 
estudiantiles que se llevan a cabo periódicamente en los institutos superiores tecnológicos del cantón Esmeraldas.

Finalmente, el artículo consta de los métodos utilizados para el diseño del software, los resultados alcanzados, una breve discusión de resultados, las conclusiones y trabajos futuros a desarrollarse a partir de la presente investigación y se termina con las referencias bibliográficas.

\section{Método}

Se utilizaron varios métodos como el analítico-sintético y el deductivo-inductivo, con el fin de extraer el valor agregado de la información recolectada y así tener una inferencia exhaustiva de los mecanismos necesarios a realizar en el desarrollo del proyecto.

Se empleó también la técnica documental para la revisión del material bibliográfico y finalmente con el uso de la técnica de la observación se realizó el seguimiento de los procesos a través de los materiales multimedia que se encuentran compartidos en internet y que dan una pauta para la realización de ciertos procesos de codificación de sitios web.

En lo referente al diseño web como tal es necesario la utilización de métodos que proporcionen la experiencia para elaborar un software con el propósito de incluir tareas de comunicación, modelación de diseño, construcción o codificación de funciones/ procedimientos y las pruebas de verificación respectivas [4].

En el desarrollo de este proyecto es necesario la utilización de una herramienta de desarrollo web para el diseño de interfaces del sistema, un motor de base de datos donde se crearon las tablas o entidades según los atributos que se emplean dentro del sistema según la categoría de usuario que acceda, además el motor de bases de datos debe permitir la determinación de relaciones entre las entidades de manera tal que los datos ingresados no tengan ambigüedad al momento de la realización de consultas por parte del administrador.

Dentro del funcionamiento de un sitio web dinámico es necesario contar con un servidor web que permite la existencia de una interfaz que establezca el punto de conexión entre el proveedor de servicios y el consumidor de servicios, de tal forma que los participantes del servicio pueden interactuar [5]. Por otro lado, la Fig. 1 muestra que los servicios ofrecidos en internet como lo es el servicio web, se basan en la relación cliente/servidor según afirma [3].

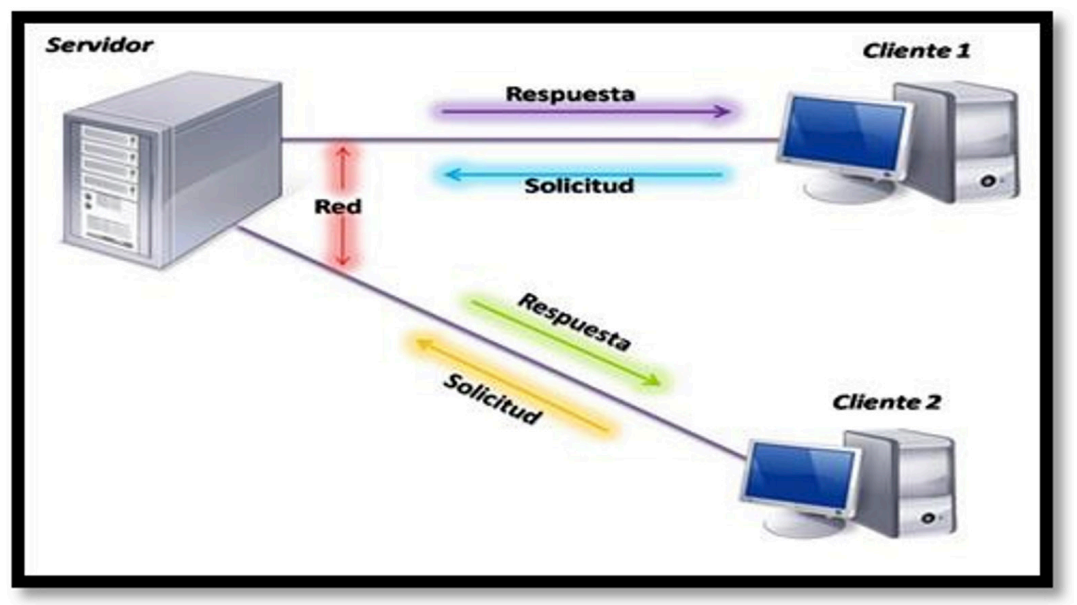

Fig. 1. Arquitectura cliente / servidor. 
Se utiliza un modelado incremental respecto a la programación del sitio debido a que este modelo aplica secuencias lineales en donde cada línea representa un incremento en el software. El modelo permite una implementación con refinamientos sucesivos y con cada incremento se agrega una nueva funcionalidad o se cubren nuevos requisitos, mejorando la versión previa implementada en el software [4].

La implementación del sitio web está estructurado en forma modular, es decir; basado en el modelo mencionado anteriormente en el que luego de seleccionar las herramientas necesarias para la implementación del sitio se inició diseñando las interfaces de tal forma que sean visualmente amigables e intuitivas, vinculando cada una de ellas de acuerdo al perfil del usuario que utilice el software. Posteriormente, como afirma [6], la construcción de una página o sitio web dinámico basado en bases de datos resulta simple con PHP, ya que este da soporte a varios tipos de bases de datos como son: Oracle, Sybase, PostgreSQL, MySQL, etc., se diseñó la base de datos constando de las entidades o tablas necesarias para alojar toda la información concerniente al sitio, estas tablas se vincularon entre sí mediante relaciones de uno a varios con el fin de evitar equívocos en los datos almacenados al momento de realizar consultas de información. Para ello, es necesario un gestor de almacenamiento como MySQL, puesto que según señala [7], es un sistema de administración de base de datos relacionales rápido, sólido y flexible.

\section{Resultados}

Al desarrollar el presente proyecto se logró la realización del diseño de las interfaces principales del sistema web, haciendo uso de la aplicación gratuita en línea Google Sites que permite a los usuarios elaborar sitios propios y de colaboración online. Además, [8] afirma que en esta aplicación se destaca la facilidad de organizar y compartir información como vídeos, imágenes, documentos, calendarios, links, etc.; además, la plataforma ofrece la posibilidad de agregar contenidos de las otras aplicaciones que brinda Google como Google Docs, Google Meet, Picasa, YouTube, etc. Así también, [9] sostiene que otras de las características de Google Sites incluyen:

- Algunas Plantillas de diseño.

- Gestión a la información con niveles de administrador, lectores y colaboradores.

- Herramienta de búsqueda con la tecnología de Google.

Las interfaces están bajo el dominio gratuito de sites.google.com ancladas entre sí desde una pantalla principal en donde el usuario según su perfil de acceso tendrá la disponibilidad de navegación en diferentes páginas. La ruta de utilización del sitio está determinada según el algoritmo mostrado en la figura 2. 


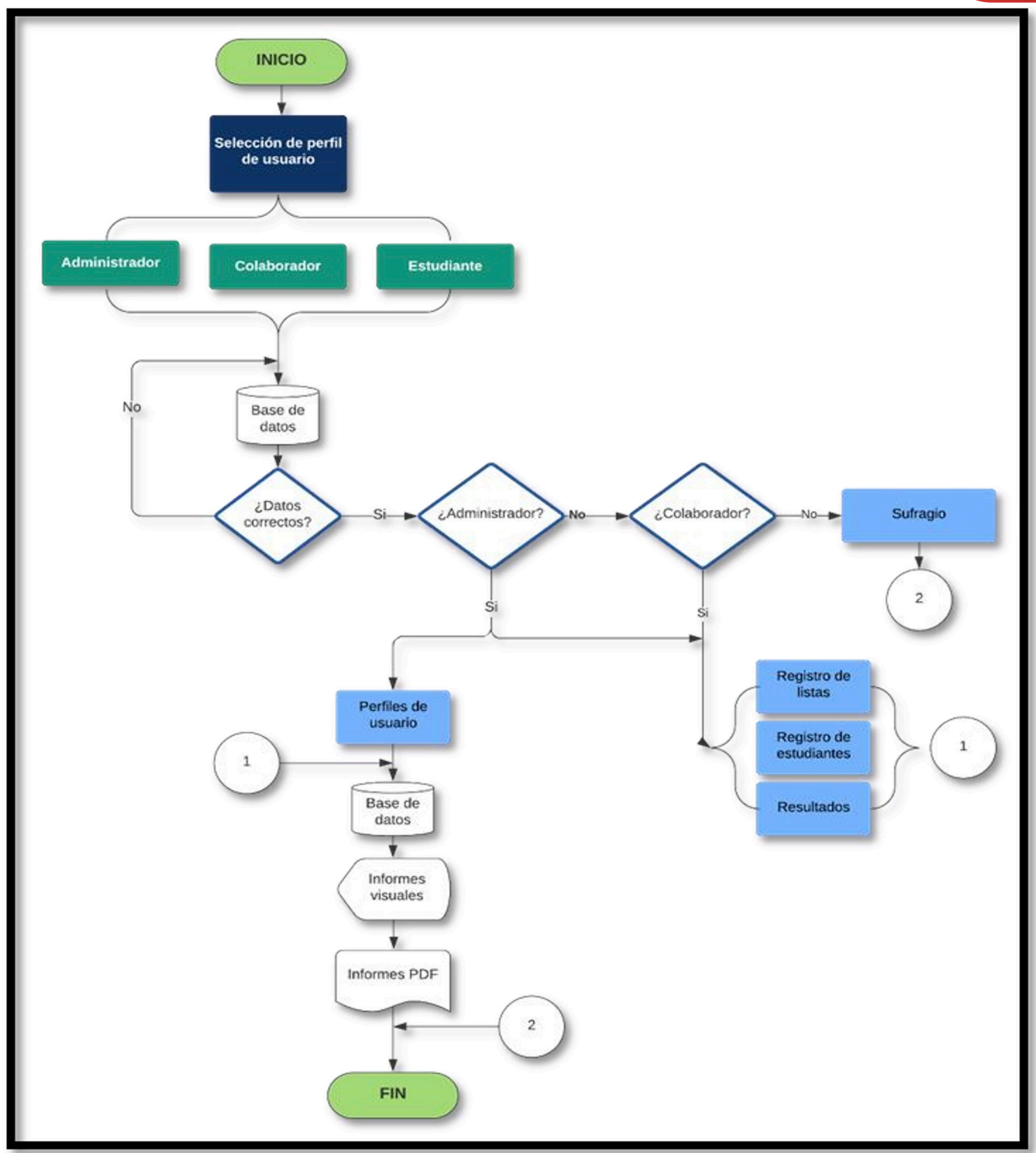

Fig. 2. Flujo de procesos del sitio web.

\section{Diseño de interfaces}

La interfaz general del sistema consistirá en una serie de páginas individuales a las que tendrán acceso los usuarios según el perfil que desempeñen dentro del sistema. Se establecieron tres tipos de perfiles de usuarios.

Primero el perfil administrador que será quien tenga acceso a todas las interfaces del sistema contando con los permisos de inserción, modificación y eliminación de datos.

Luego está el perfil de colaborador que es quien tenga el permiso de insertar datos en ciertas páginas según lo haya establecido el administrador del sistema.

Finalmente, el sistema contará con el perfil de estudiante que tendrá permiso de inserción de datos en la única página a disposición. Las funcionalidades, permisos y páginas de acceso se detallan en la tabla 1. 
Tabla 1. Funcionalidades y permisos de usuarios.

Páginas sitio

\begin{tabular}{|l|l|l|l|}
\hline Registro de usuarios & Lectura/escritura & Lectura/escritura & \\
\hline Registro de listas & Lectura/escritura & Lectura/escritura & \\
\hline Informe de resultados & Lectura/escritura & Lectura & Lectura \\
\hline Perfiles de acceso & Lectura/escritura & & \\
\hline Configuración & Lectura/escritura & & \\
\hline Sufragio & & & Lectura/escritura \\
\hline \multicolumn{1}{|c|}{ Perfiles } & Administrador & Colaborador & Estudiante \\
\hline
\end{tabular}

Las interfaces diseñadas para el sitio web fueron realizadas según los procesos principales con los que cuenta el sistema. En referencia a la ruta de navegación del sistema, se tiene como pantalla inicial la pantalla de perfiles de usuario (véase Fig. 3), en donde el usuario al momento de autenticarse mediante su nombre de usuario y contraseña accederá a diferentes páginas según sea el perfil registrado en la base de datos del sistema; este proceso de verificación de acceso se hará mediante la codificación del sitio.

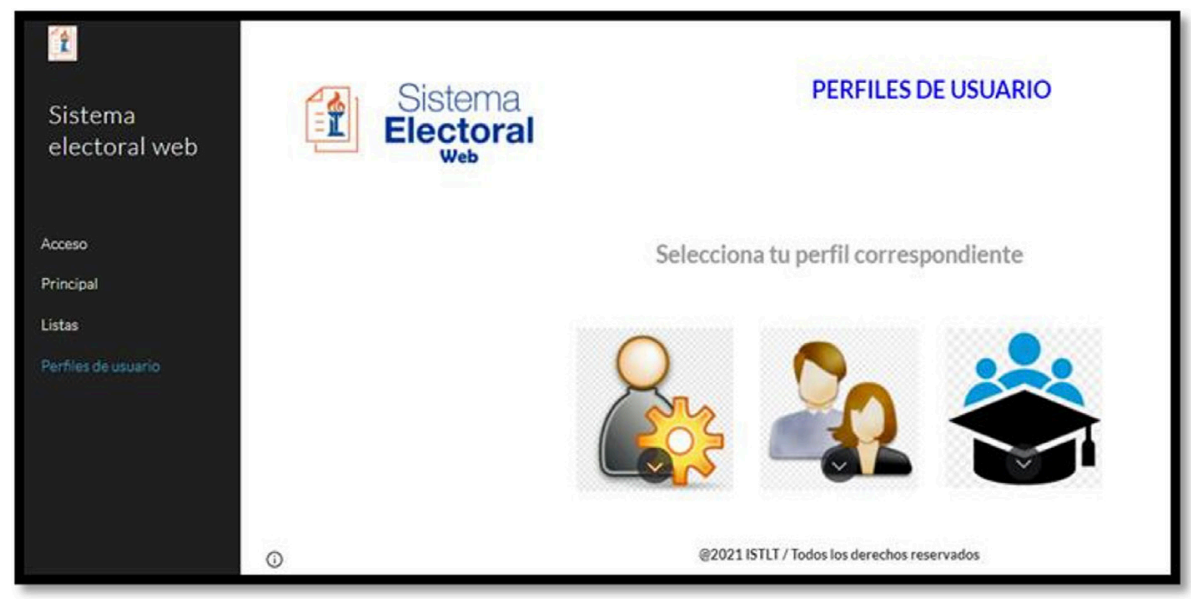

Fig. 3. Pantalla de perfil de usuario.

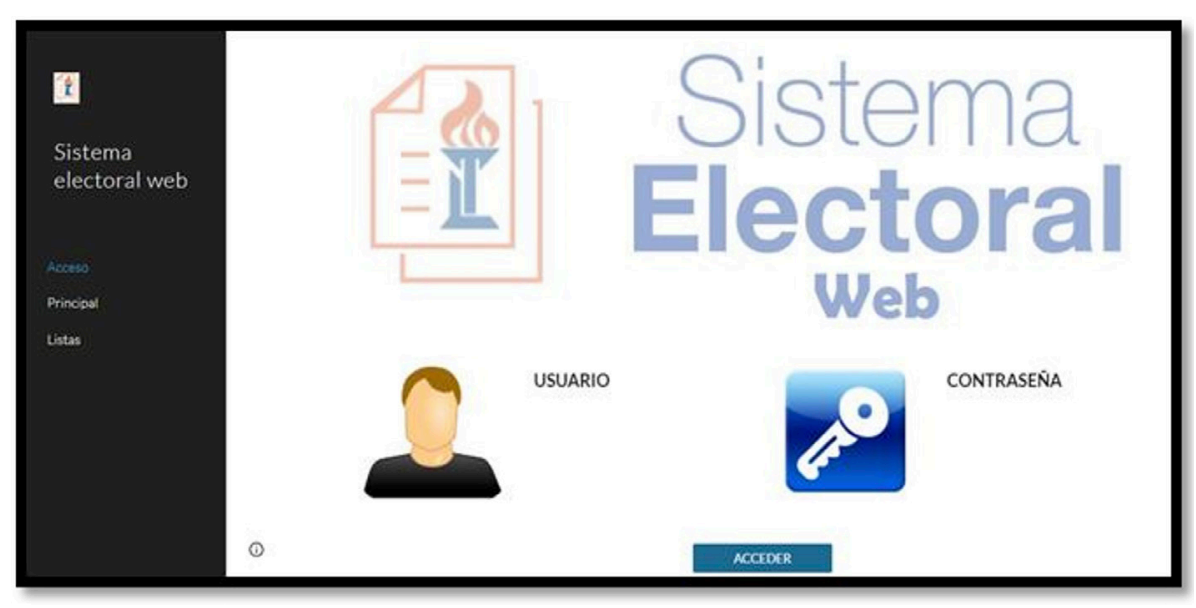

Fig. 4. Pantalla de acceso principal.

Si el usuario corresponde a un estudiante, éste accederá a la pantalla principal donde se 
visualizarán dos opciones: sufragio e información de listas. La primera corresponde a la interfaz donde se llevará a cabo la votación entre las listas registradas para el proceso de elecciones, la segunda interfaz hace referencia a información de los integrantes de las listas del proceso de elecciones en donde se visualizarán los datos de los integrantes (véase Fig. $5,6$ y 7$)$

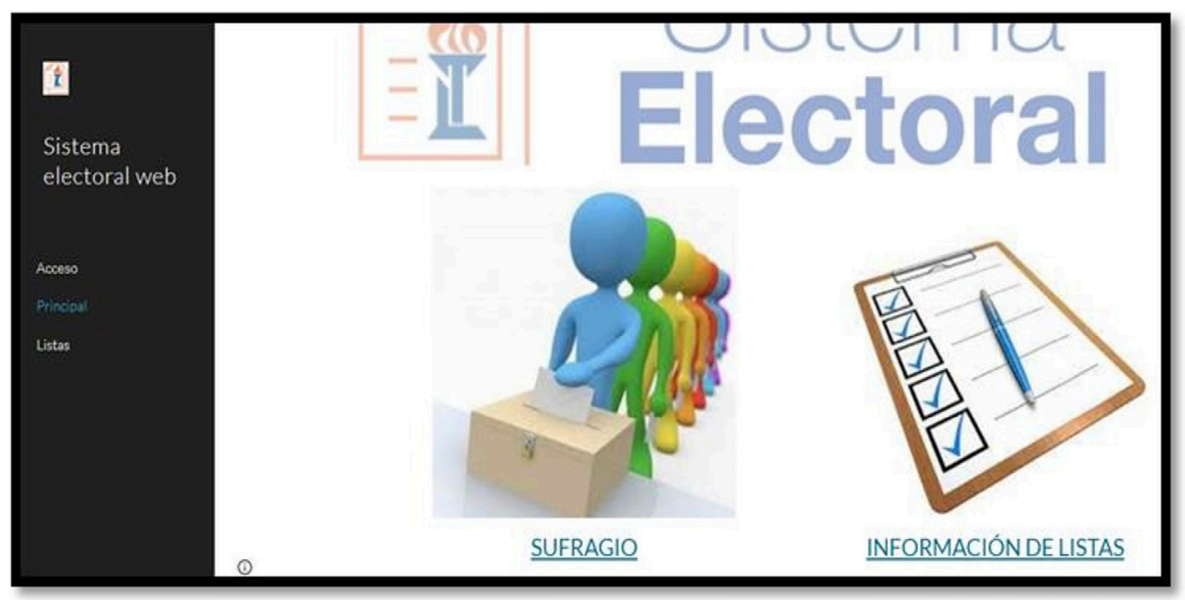

Fig. 5. Pantalla principal estudiantes.

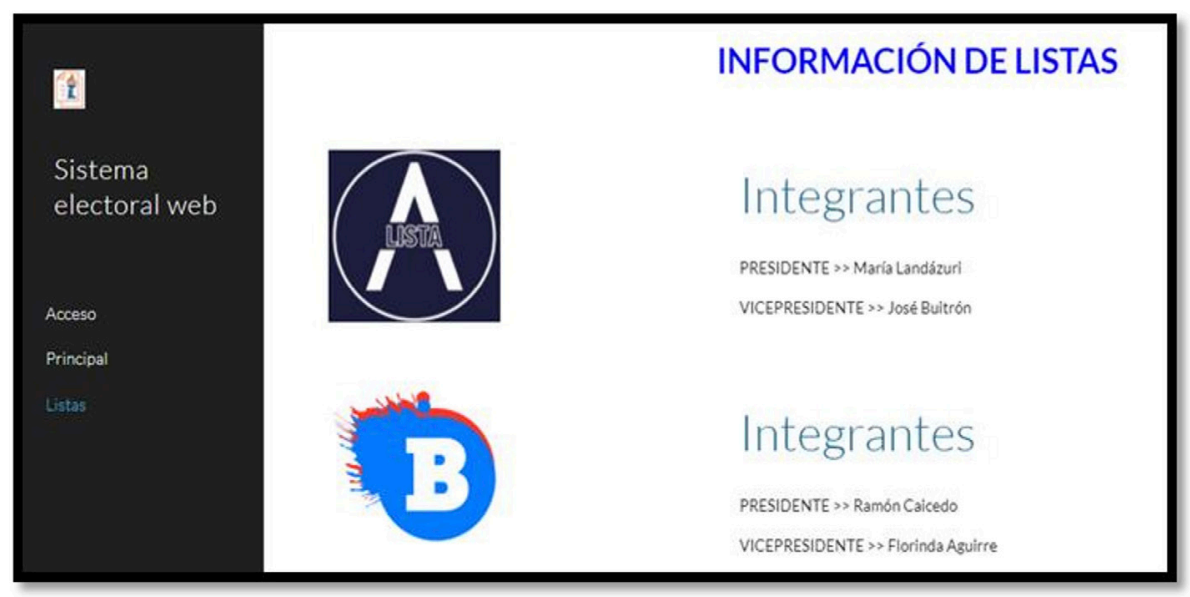

Fig. 6. Pantalla información de listas.

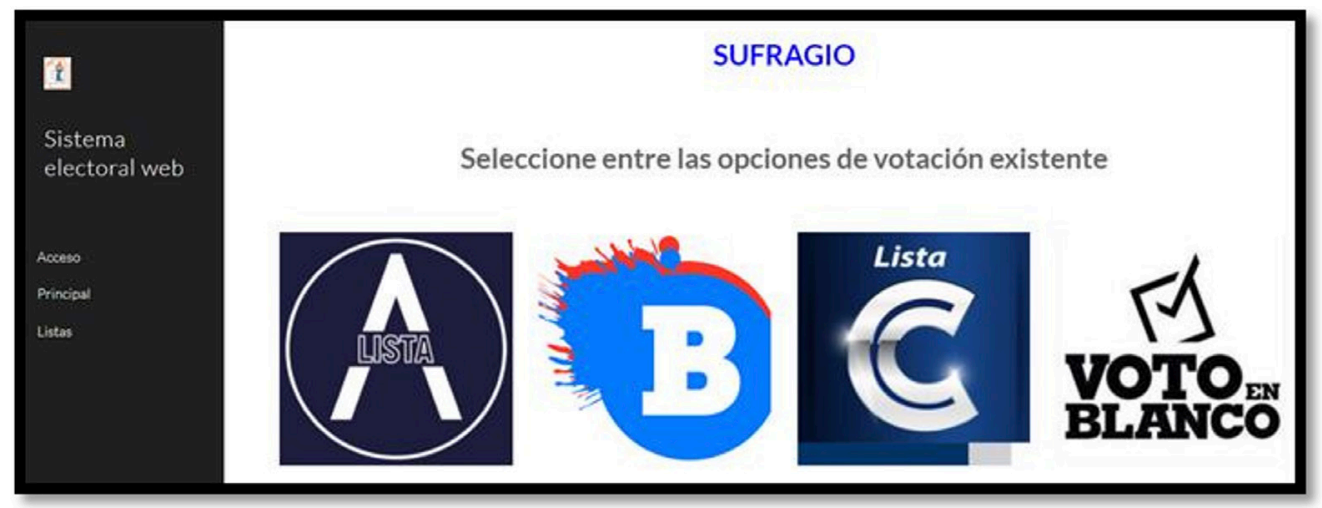

Fig. 7. Pantalla de sufragio. 
Por otro lado, si el usuario que accede al sitio es un colaborador según la autenticación del sistema, éste tendrá la disponibilidad de páginas que permitirán el registro de datos de estudiantes, listas, además de visualizar el informe de resultados del proceso de elecciones (véase Fig. 8).

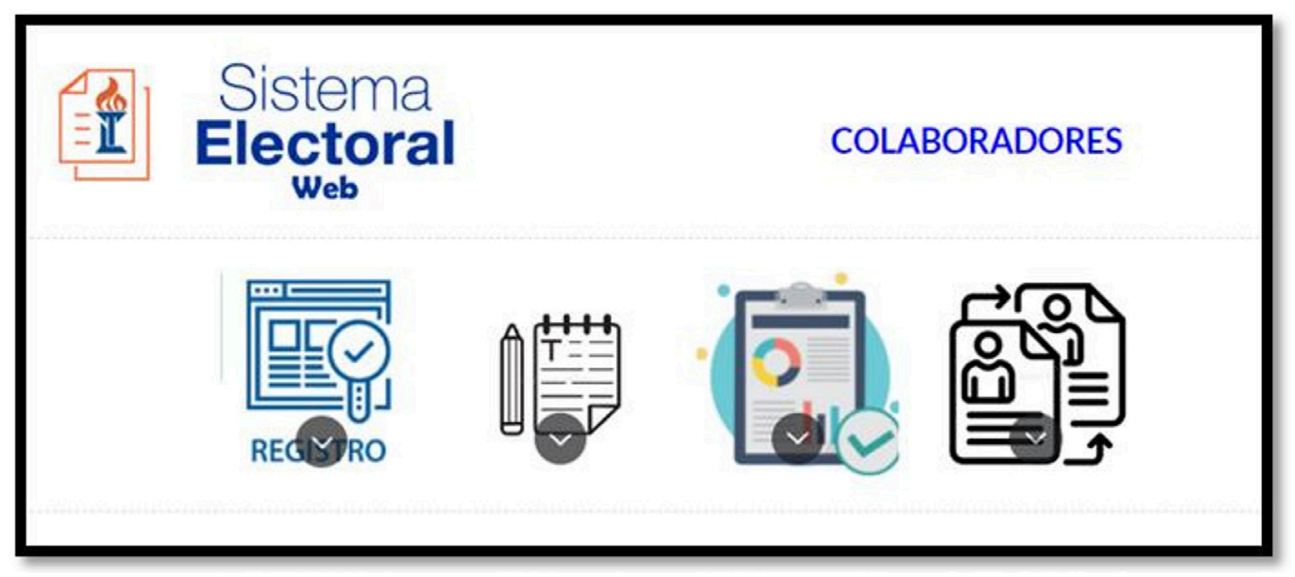

Fig. 8. Pantalla principal colaboradores.

En la pantalla de registro de estudiantes se ingresarán todos los datos personales para ser almacenados por curso, paralelo y carrera en las entidades de la base de datos (véase Fig. 12). En la pantalla de registro de listas, se ingresarán los integrantes que conforman la lista respectiva. Estos datos ya deben estar almacenados previamente en la base de datos, puesto que ningún miembro de una lista puede no pertenecer a algún curso o carrera del instituto. Para esto la interfaz cuenta con un botón de búsqueda que permitirá consultar en la base de datos mediante un campo clave el estudiante a ser registrado en la lista, y una vez encontrado el estudiante éstos datos se cargarán automáticamente para ser almacenados en la entidad respectiva (véase Fig. 13).

Dentro del perfil de colaborador también está disponible la pantalla de visualización de resultados. Debido a que es un sistema informático y está online, el conteo de los votos es automático y podrán ser visualizados en este perfil mediante la pantalla de resultados parciales. Estos datos se irán actualizando conforme los estudiantes registren sus respectivos sufragios dentro del periodo estipulado por parte del ente regulador del proceso. Cabe señalar que en este perfil no se podrán realizar modificaciones o eliminaciones de registros en las pantallas anteriores (véase Fig. 9).

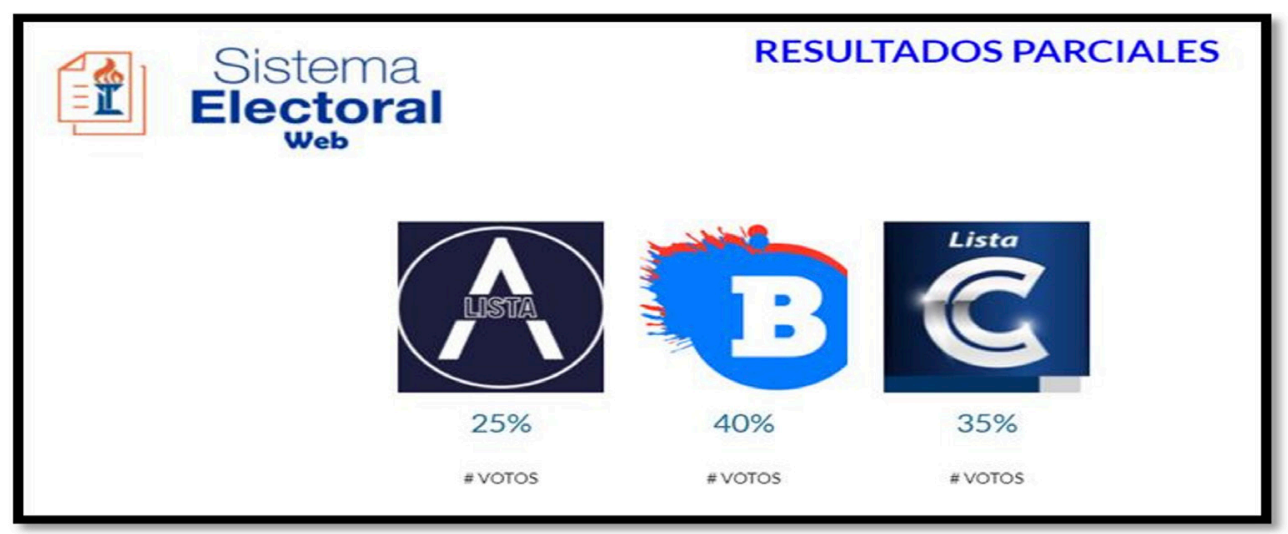

Fig. 9. Pantalla de resultados parciales. 
Finalmente el perfil dispone de la pantalla de actualización de datos donde el colaborador podrá realizar el cambio de la información de su perfil como nombres, apellidos y contraseña (véase Fig. 10).

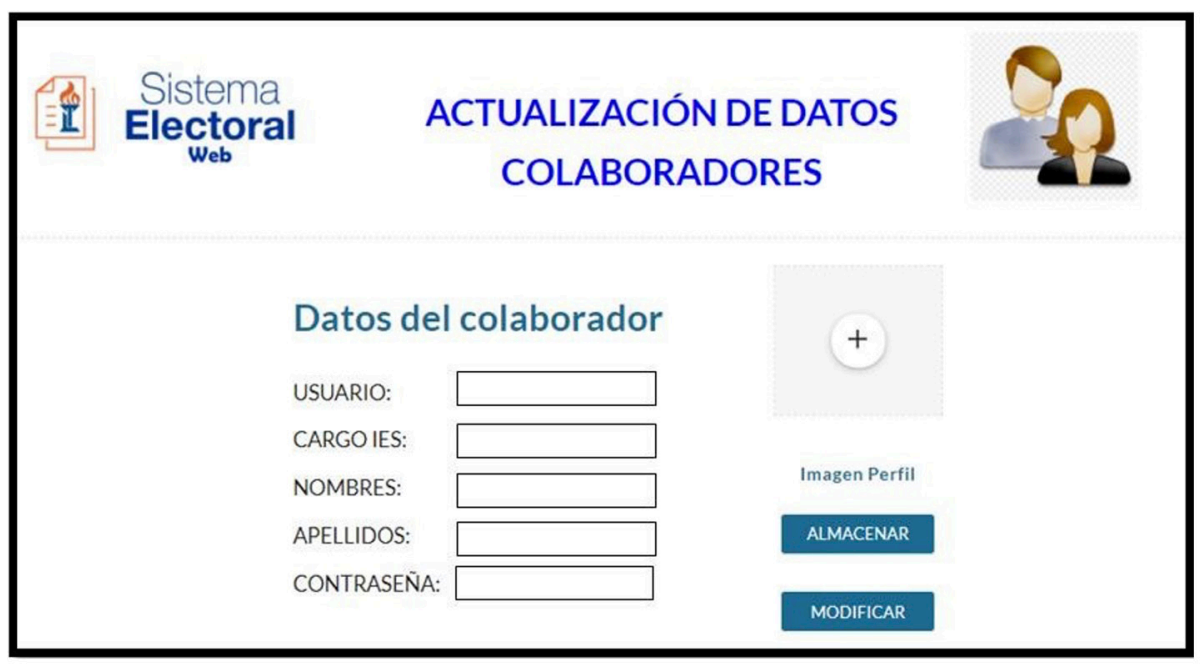

Fig. 10. Pantalla actualización de datos.

En lo referente al perfil administrador se destaca que es el usuario con todos los permisos sobre la información almacenada en la base de datos del sistema web, así como también la disponibilidad de acceso a las mismas pantallas que el perfil de colaborador con la diferencia de poder hacer modificaciones y/o eliminaciones de datos (véase Fig. 11).

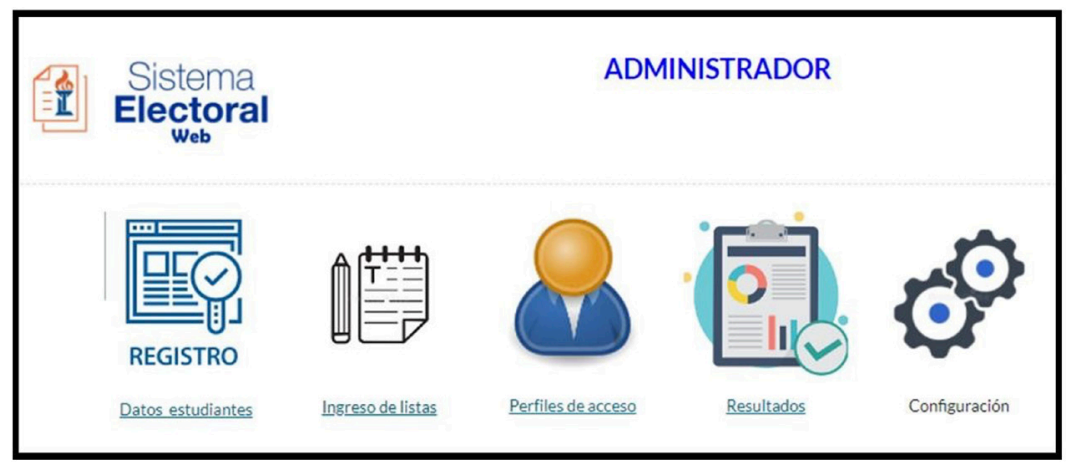

Fig. 11. Pantalla principal administrador.

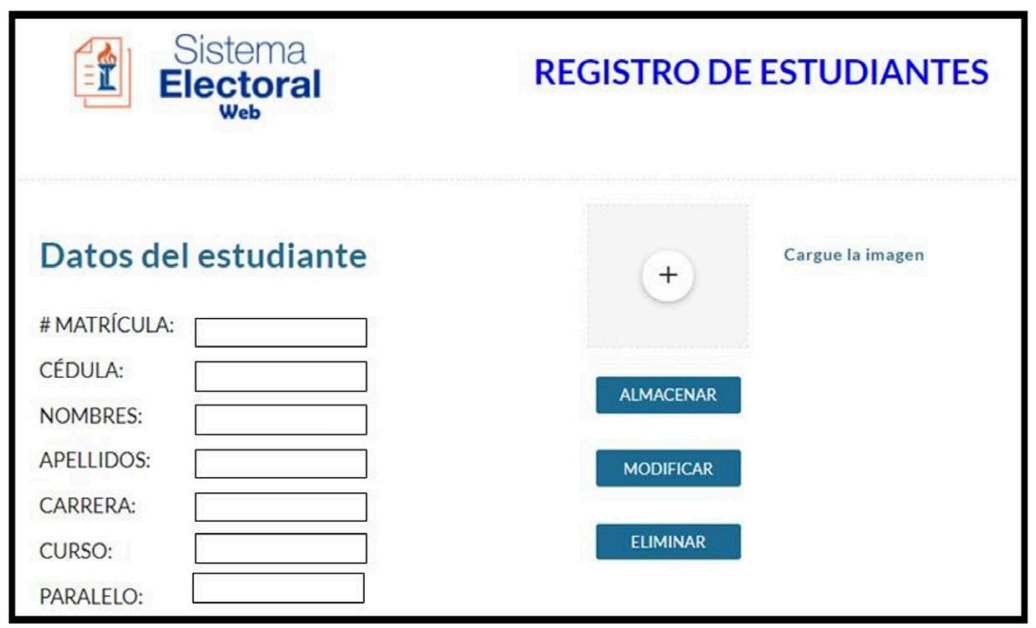

Fig. 12. Pantalla de registro de estudiantes. 


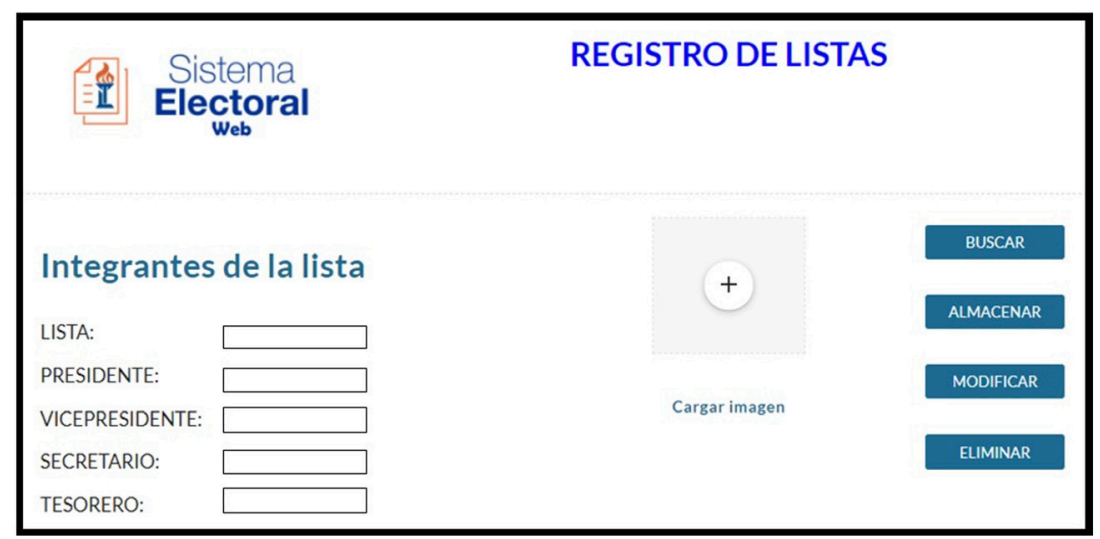

Fig. 13. Pantalla de registro de listas.

Por otro lado, este perfil dispondrá de la pantalla de perfiles en donde se podrán registrar nuevas cuentas de colaboradores del sistema (véase Fig. 14). Las credenciales de acceso se establecerán automáticamente según los datos ingresados.

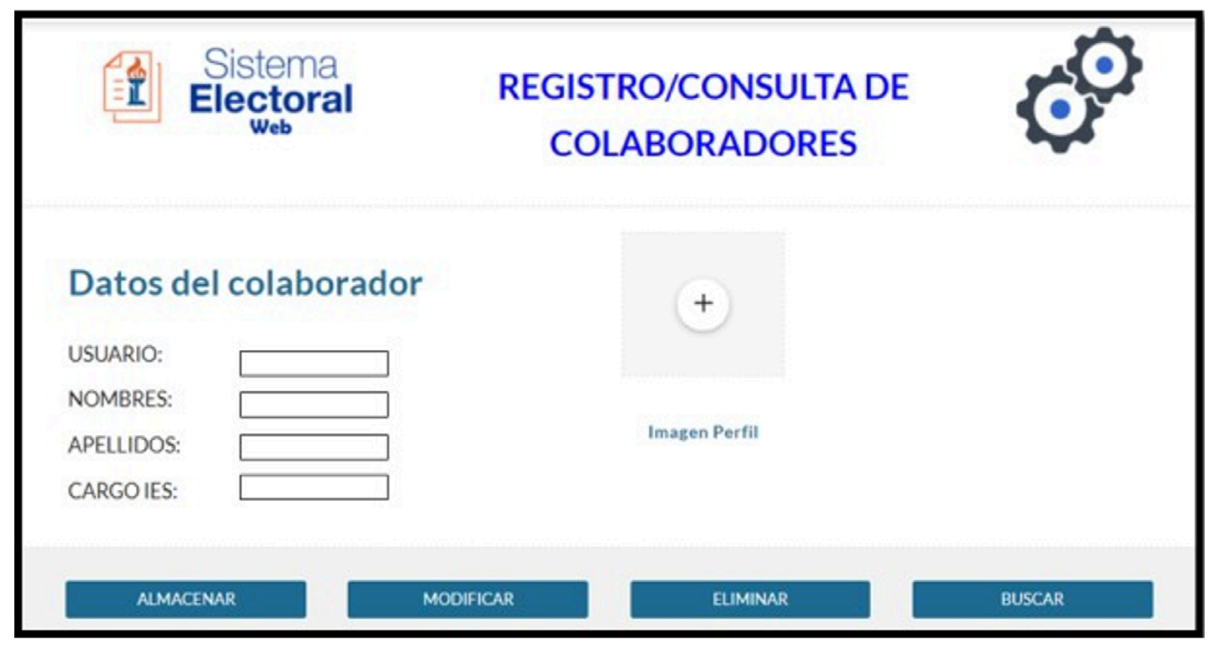

Fig. 14. Pantalla de consulta de colaboradores.

\section{Diseño de base de datos}

La base de datos a implementar en el sistema web fue diseñada con un motor de base de datos local en donde se establecieron las diferentes entidades con sus atributos respectivos. La mayor parte de las entidades o tablas constan con un campo clave que permitió vincular o relacionarlas entre sí con el propósito de no crear datos duplicados y por consiguiente valores ambiguos en la búsqueda de información.

El modo de relación entre las tablas es de uno a varios, partiendo del campo único en las tablas principales hasta aquellos campos en que sus valores pueden ser repetitivos a lo largo de la funcionalidad del sistema web. Las tablas diseñadas y las relaciones entre ellas se visualizan en las figuras siguientes. 


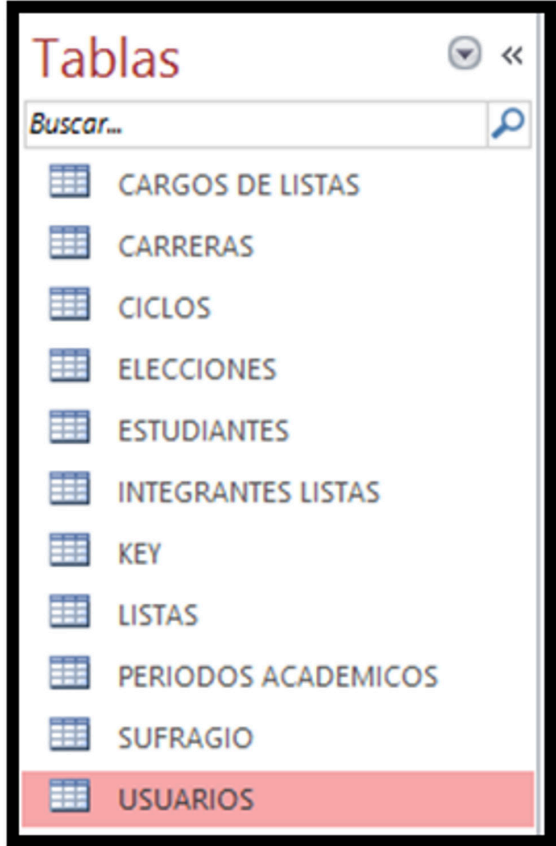

Fig. 15. Entidades de la base de datos.

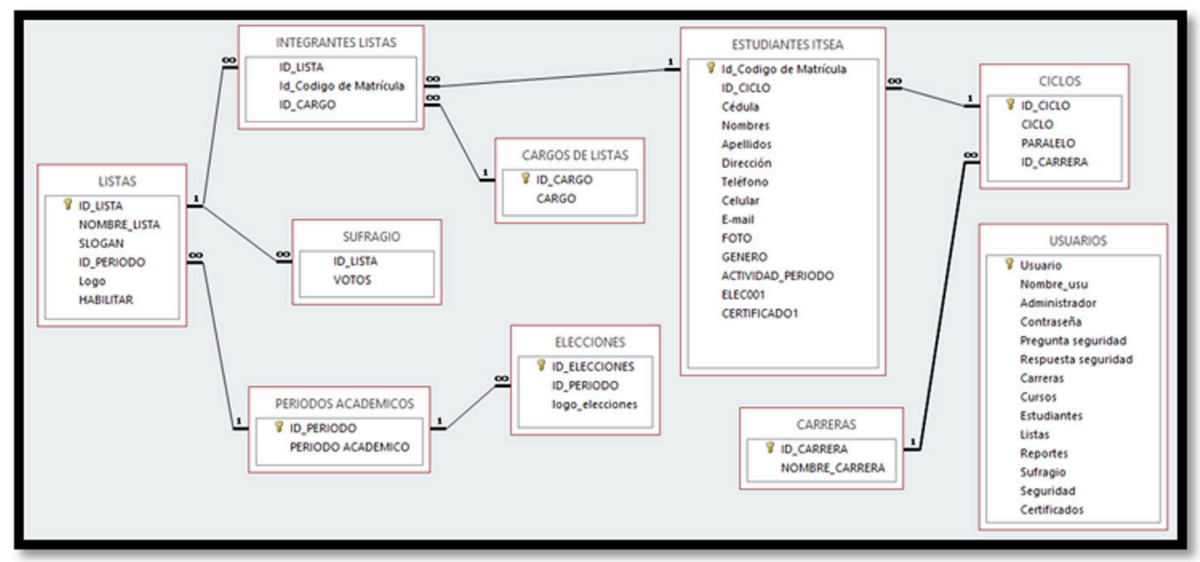

Fig. 16. Relaciones entre entidades.

\section{Discusión de resultados}

En referencia a la eficacia de comunicación que brindaría la implementación de un sitio web para el proceso de elecciones estudiantiles en el ISTLT, [10] señala que la web en la actualidad es un instrumento que permite perfeccionar la democracia rompiendo el modelo unidireccional de comunicación, ayudando a que los sufragantes puedan verter su criterio respecto a propuestas mediante redes sociales, sintiéndose en ese momento parte del proceso.

Con la implementación de un sitio web se logrará que aquellos estudiantes que por factores ajenos a su voluntad como el empleo, tiempo, distancia, etc. no podían ejercer su voto físico en el horario dispuesto por el ente regulador, tengan la libertad de ser partícipes del proceso tomando un momento del día, desde el lugar y comodidad donde se encuentre para ingresar desde su dispositivo conectado al internet a la plataforma y ejercer su voto. 
Por otro lado, en el ámbito de la comunicación a través del internet se destaca la credibilidad de páginas o sitios web que operan a diario en esta red global, generando cierta suspicacia por parte de los usuarios en que la información que se visualiza pueda tener un alto porcentaje de falsedad; respecto a lo anteriormente mencionado [11] enfatiza que la credibilidad de los sitios web deben estar sujetos a sistemas de control con el fin de conseguir con precisión las respuestas obtenidas desde el internet. En este contexto se presentan varios módulos inherentes a los sistemas de control como la calidad, la reputación, originalidad, frecuencia de actualización etc., como también el uso de ciertas interfaces de programación de aplicaciones (API) y técnicas de procesamiento de información se mejora la puntuación de credibilidad de los sitios web (véase Fig. 17).

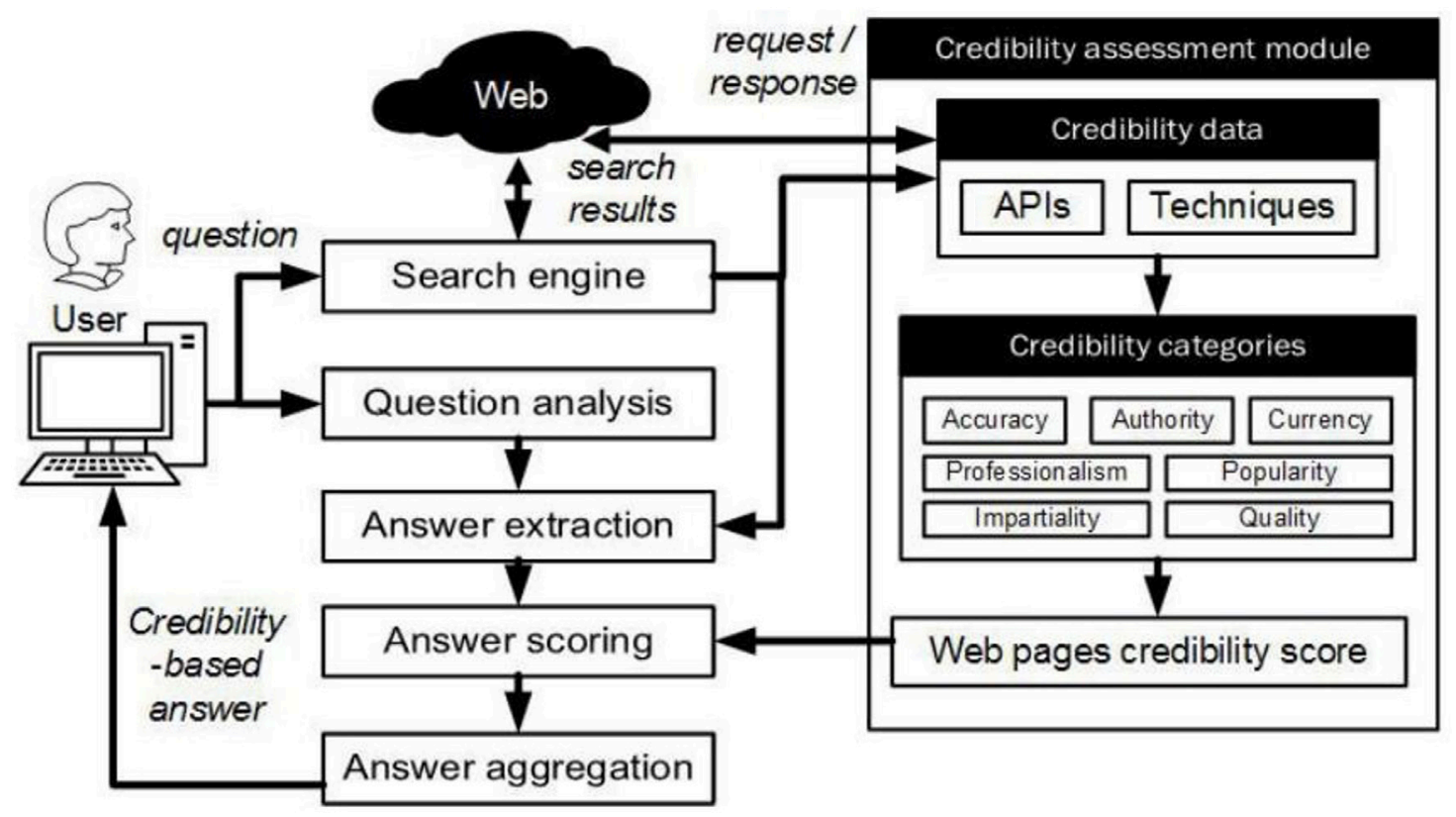

Fig. 17. Generar respuestas basadas en credibilidad, utilizando un módulo de evaluación de credibilidad. Fuente [11].

La implementación de un sistema web para los procesos de elecciones estudiantiles difiere respecto a la credibilidad de los sitios web manifestados anteriormente ya que en ese aspecto en particular se basan en sitios web que proporcionan búsquedas masivas de información en donde en pocos segundos se encuentran cientos o miles de resultados sin que el usuario final conozca la veracidad de la información obtenida; el proceso que llevará a cabo el sistema propuesto no está basado en motores de búsqueda sino más bien en la codificación exclusiva del sitio para el proceso de elecciones, donde cada línea de comando utilizada buscará satisfacer la funcionalidad eficaz del sistema vinculado a las interfaces y base de datos diseñadas para el almacenamiento de la información. Por otra parte, es necesario resaltar que el acceso al sitio web no solo estará restringido mediante las autenticaciones comunes usuario y contraseña que todo sistema informático debe tener sino que dichos usuarios pertenecerán íntegramente a la institución, descartando así la masiva utilización del sitio. Finalmente la información que viaje en la red hasta el almacenamiento remoto en la base de datos contará con la encriptación de datos que proporcionan por defecto los servidores de alojamiento de sitios web a nivel mundial. 


\section{Conclusiones y trabajos futuros}

- La búsqueda de información en el desarrollo del proyecto vislumbró que existen varias investigaciones que encajan perfectamente en el contexto de la problemática presentada y sustentan que el uso del internet en medio de procesos electorales mejora la comunicación y empoderamiento de los participantes con el proceso al no tener la obligatoriedad de traslado físico. Toda esta sustentación teórica encontrada aportó considerablemente en la gestación del proyecto, ya que dio luces y ánimo investigativo sabiendo que la temática propuesta no está enlazada a una idea descabellada o infructuosa para su investigación.

- La herramienta Google Sites para el diseño del sitio permitió facilidad de uso, gratuidad del servicio y presentación amigable e intuitiva de las interfaces, en las que de manera estructurada se jerarquizaron las distintas páginas de acceso según los perfiles de usuario plasmados. Además resultó necesario que las páginas cuenten con elementos primordiales como imágenes, cajas de texto, cuadros combinados, botones, etc. que permitirán el ingreso y presentación de información hacia y desde la base de datos respectivamente como también la vinculación entre las diferentes páginas del sitio.

- El almacenamiento de información del sitio web diseñado no tendrá dificultades de acceso ni tampoco ambigüedades en los datos ya que los mismos estarán relativamente indexados gracias a la creación de entidades con los atributos correspondientes que alojarán cada registro ingresado por los usuarios del sistema. Conjuntamente a ello se establecieron relaciones de tipo uno a varios entre las entidades para disponer del acceso inmediato de la información mediante consultas según los requerimientos de uso, brindando así un conjunto compacto de información en la que el sistema podrá acceder remotamente a los datos desde una tabla a cualquier otra mediante la conexión de sus campos claves, todo esto desde un trabajo en segundo plano invisible para el usuario final.

- Las interfaces del sitio web no cuentan con programación que defina su funcionamiento completo puesto que la herramienta utilizada en la creación de las interfaces es básica y no soporta codificaciones en lenguajes como HTML, CSS o JavaScript sino que proporciona un aspecto intuitivo de navegación. Por tal razón el presente desarrollo sirve de base para la realización de proyectos más amplios y completos que incluyan el diseño y programación con herramientas más sofisticadas y profesionales en la interacción de contenido web dinámico. En tal efecto si se continúa el proyecto presentado se debe pretender desarrollar la programación de cada página del sitio con comandos propios de la herramienta de codificación y luego vincular la base de datos con la interfaz del sitio de modo que los datos insertados por el usuario se almacenen en las tablas o entidades correspondientes según la relación existente entre ellas. Finalmente se deberían realizar las verificaciones de funcionamiento desde el ingreso de datos hasta los informes de resultados finales. 


\section{Referencias}

D. Calvo, E. Campos-Domínguez, and M. Díez-Garrido, "Hacia una campaña computacional: herramientas y estrategias online en las elecciones españolas," Revista Española de Ciencia Política, vol. 51, pp. 123-154, 2019.

M. Z. Espinosa, S. E. Balseca, and C. R. Valenzuela, "EL USO DE LAS PLATAFORMAS Facebook, Twitter y Whatsapp COMO MEDIO DE PARTICIPACIÓN POLITICA Online EN LOS PROCESOS," Boletín académico Sociol. Y POLÍTICA HOY, no. 5, pp. 135-146, 2021, [Online]. Available: https://revistadigital.uce.edu.ec/index.php/hoy/ article/view/3254/3802.

E. Condor and I. Soria, Programación Web con CSS, JavaScript, PHP y AJAX - Enrique E. Condor Tinoco, Iván Soria Solís - Google Libros. 2014.

F. José and G. Peñalvo, Ingeniería de Software I. 2019.

I. Zafar, F. Azam, M. W. Anwar, B. Maqbool, W. H. Butt, and A. Nazir, "A novel framework to automatically generate executable web services from bpmn models," IEEE Access, vol. 7, pp. 93653-93677, 2019, doi: 10.1109/ACCESS.2019.2927785.

M. Arias, Aprende Programación Web con PHP y MySQL: 2a Edición - Miguel Ángel Arias - Google Libros, 2da. IT Campus Academy, 2017.

Á. Cobo, PHP y MySQL: Tecnología para el desarrollo de aplicaciones web. - Ángel Cobo - Google Libros. Ediciones Díaz de Santos, 2005.

C. Herrra, "Ventajas y desventajas de Google Sites para un blogger - Bloguero Pro," Entiende la tecnología, 2020. https://blogueropro.com/blog/ventajas-y-desventajasdegoogle-sites-para-un-blogger (accessed Oct. 22, 2021).

WebGratis.page, "¿Qué es Google Sites?," 2021. https://webgratis.page/que-esgooglesites/ (accessed Oct. 22, 2021).

F.-A. Cariola and P. Gavilán, "Eficacia de los sitios webs como herramienta comunicacional de los Diputados chilenos. Efficacy of websites as a communication tool for Chilean deputies," Revista de Comunicación, vol. 18, no. 2, pp. 139-154, 2019.

A. A. Shah, S. D. Ravana, S. Hamid, and M. A. Ismail, "Web Pages Credibility Scores for Improving Accuracy of Answers in Web-Based Question Answering Systems," IEEE Access, vol. 8, pp. 141456-141471, 2020. 